\title{
Application of uniform design to improve dental implant system
}

\author{
Yung-Chang Cheng ${ }^{\mathrm{a},{ }^{*}, \text { Deng-Huei Lin }{ }^{\mathrm{a}} \text { and Cho-Pei Jiang }}{ }^{\mathrm{b}}$ \\ ${ }^{a}$ Department of Mechanical and Automation Engineering, National Kaohsiung First University of \\ Science and Technology, Taiwan \\ ${ }^{b}$ Department of Power Mechanical Engineering, National Formosa University, Taiwan
}

\begin{abstract}
This paper introduces the application of uniform experimental design to improve dental implant systems subjected to dynamic loads. The dynamic micromotion of the Zimmer dental implant system is calculated and illustrated by explicit dynamic finite element analysis. Endogenous and exogenous factors influence the success rate of dental implant systems. Endogenous factors include: bone density, cortical bone thickness and osseointegration. Exogenous factors include: thread pitch, thread depth, diameter of implant neck and body size. A dental implant system with a crest module was selected to simulate micromotion distribution and stress behavior under dynamic loads using conventional and proposed methods. Finally, the design which caused minimum micromotion was chosen as the optimal design model. The micromotion of the improved model is $36.42 \mu \mathrm{m}$, with an improvement is $15.34 \%$ as compared to the original model.
\end{abstract}

Keywords: Zimmer implant, dynamic loads, micromotion, uniform design

\section{Introduction}

Dental implants have been widely and effectively used in recent years to substitute for missing teeth, primarily due to their restoring stability. A periodic loading has been proven to raise the primary stability and decrease the failure rate of dental implants. Javed and Romanos [1] studied the various results of primary implants caused by poor osseointegration, weak biomechanical properties at the osteotomy site, or biomechanical overloading. Occlusal overload has been proven to result in marginal bone loss around dental implants, with no inflammation in the peri-implant and a complete or partial absence of osseointegration [2]. Kayabasi, et al. [3] investigated the stresses and strains on dental implant systems by using finite element analysis. Kim and Shin [4] revealed the effects of three abutment types and dynamic loading on the stability of implant prostheses. Mammadzada, et al. [5] investigated the effect of abutment and implant shapes on stresses in dental applications. Employing the finite element method, much previous literature has presented the loading effect on dental implant system. Most research has studied the effect of static loadings on the behavior of the dental implant system. However, the forces acting on the dental implant system are dynamic loadings, making the

\footnotetext{
* Address for correspondence: Yung-Chang Cheng, Department of Mechanical and Automation Engineering, National Kaohsiung First University of Science and Technology, Taiwan. Tel.: +886-7-6011000 ext. 2293; Fax: +886-7-6011066; Email: yccheng@nkfust.edu.tw.
} 
numerical analysis of static loads is unsuitable for the study of dental implant systems.

This paper presents the innovative integration of explicit dynamic finite element analysis and uniform experimental design for the Zimmer dental implant. First, uniform experimental design is applied to generate a group of experiments. Next, ANSYS/LS-DYNA software is utilized to evaluate micromotions of cortical and cancellous bones to simulate the action of the dynamic chewing load on the implant model. Finally, uniform design is applied to experimental simulations to minimize micromotion of the implant model.

\section{Analysis method}

\subsection{Dental implant system}

The 3-dimensional model and geometric design of a Zimmer dental implant is constructed using Pro/Engineer software as shown in Figure 1. The dimensional characteristics of the Zimmer implant are listed in Table 1: (A) thread pitch, (B) body size, (C) thread depth, (D) maximum diameter of the implant, (D1) larger diameter of the implant neck and (D2) smaller diameter of the implant neck. Figure 2 illustrates the key components of the dental implant: implant, cortical bone, and cancellous bone [6].

\subsection{Explicit dynamic finite element analysis}

HyperMesh meshing software was utilized to create the 3-dimensional models in the preprocess analysis. The contact conditions between the implant and bone are given to evaluate the mechanics at the implant-bone interface (Figure 3). Theses interfaces are frictional surfaces of which the frictional coefficient is equal to 0.3 , before osseointegration [7]. The boundary conditions for the 3-dimensional models are fixed in all directions on the mesial and distal surfaces.

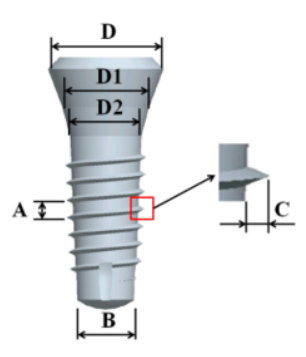

Fig. 1. 3-dimensional model of Zimmer implant.

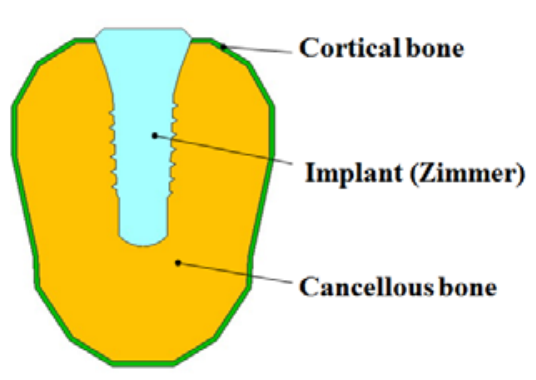

Fig. 2. Dental implant system.

Table 1

Geometric dimensions of implant

\begin{tabular}{llllll}
\hline $\begin{array}{l}\text { Thread } \\
\text { pitch }\end{array}$ & $\begin{array}{l}\text { Body } \\
\text { size }\end{array}$ & $\begin{array}{l}\text { Thread } \\
\text { depth }\end{array}$ & $\begin{array}{l}\text { Maximum diameter } \\
\text { of implant neck }\end{array}$ & $\begin{array}{l}\text { Larger diameter } \\
\text { of implant neck }\end{array}$ & $\begin{array}{l}\text { Smaller diameter } \\
\text { of implant neck }\end{array}$ \\
A $(\mathrm{mm})$ & B $(\mathrm{mm})$ & C $(\mathrm{mm})$ & D $(\mathrm{mm})$ & D1 $(\mathrm{mm})$ & D2 $(\mathrm{mm})$ \\
\hline 0.9 & 2.98 & 0.33 & 6.0 & 4.5 & 3.7 \\
\hline
\end{tabular}




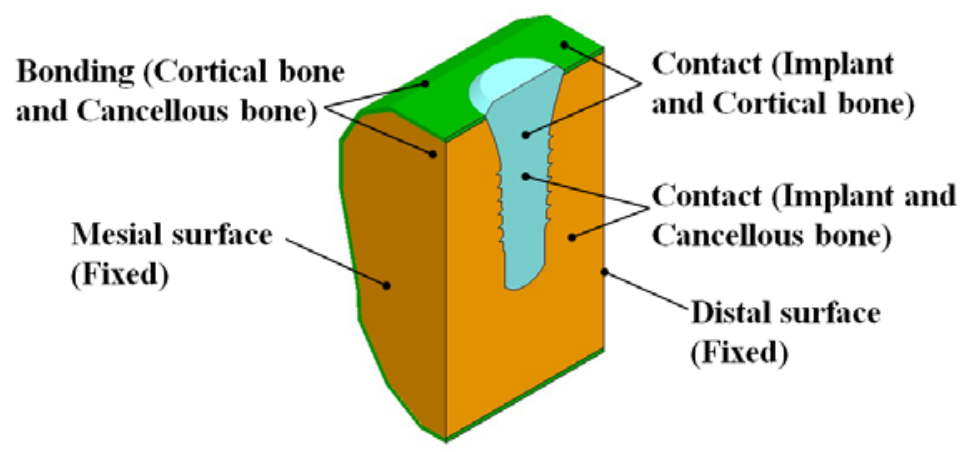

Fig. 3. Boundary settings of finite element model.

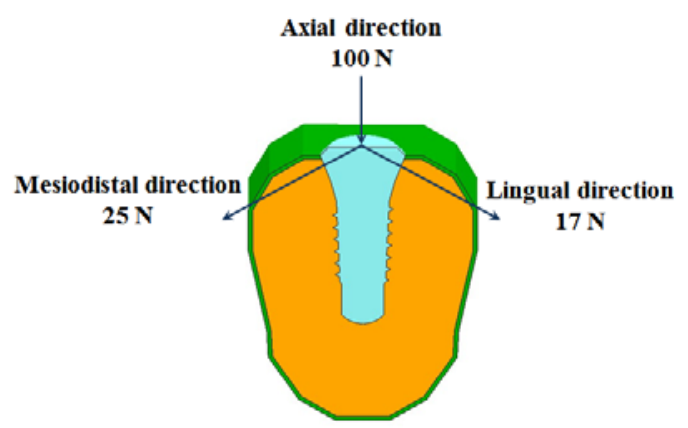

Fig. 4. Components of implant load.

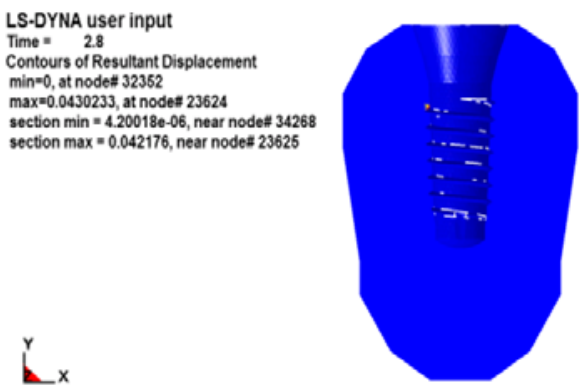

(a)

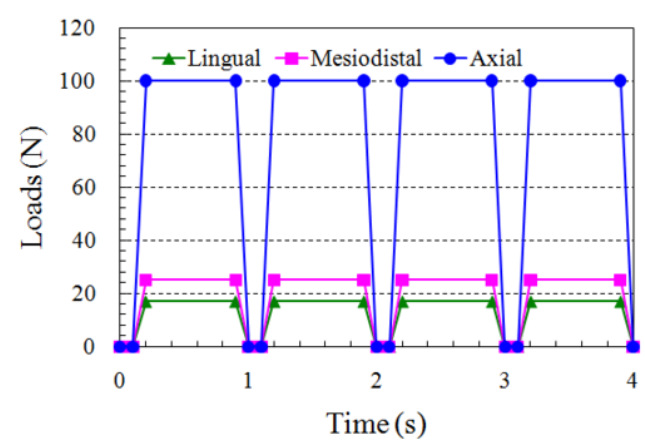

Fig. 5. Dynamic loads behavior.

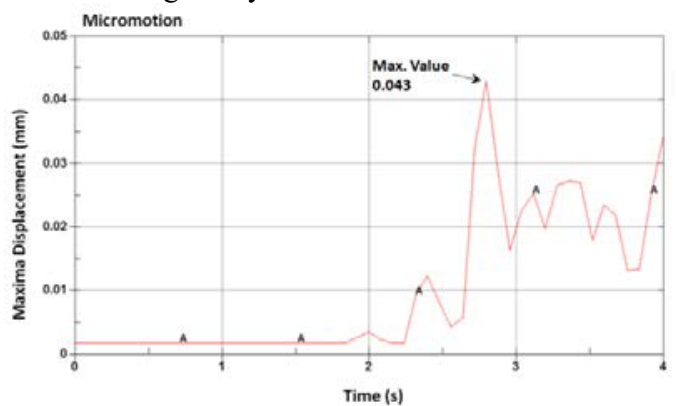

(b)

Fig. 6. (a) Contours and (b) dynamic response of micromotion of dental implant system.

In this paper, the loads acting on the implant settings are categorized as dynamic. Loads are assumed to act in three directions, as shown in Figure 4 [6]. In addition, the distributions of dynamic loads are presented in Figure 5, to simulate the actual chewing situation.

The mechanical properties of bones differ with bone quality and age of the patient. In this study, Young's modulus and Poisson's ratio for the implant, cortical layer, and cancellous layer are assumed to be constant, as shown in Table 2 [6]. The implant is a titanium alloy, Ti6A14V [6]. All materials in the simulation analysis are homogeneous, isotropic and linearly elastic

Micromotion occurs in the district of the per-implant as loads acting on the implant, which may interfere with the process of osseointegration. The amount of micromotion is an important issue that largely determines the success rate of implantation. For the Zimmer implant model, the maximum 
Table 2

Material characteristic settings in finite element analysis [6]

\begin{tabular}{llll}
\hline Material & Poisson's ratio & Young's modulus E $(\mathrm{GPa})$ & Density $\left(\mathrm{mg} / \mathrm{cm}^{3}\right)$ \\
\hline Implant & 0.35 & 110 & 4500 \\
Cortical bone & 0.3 & 13 & 2400 \\
Cancellous bone & 0.3 & 0.345 & 1100 \\
\hline
\end{tabular}

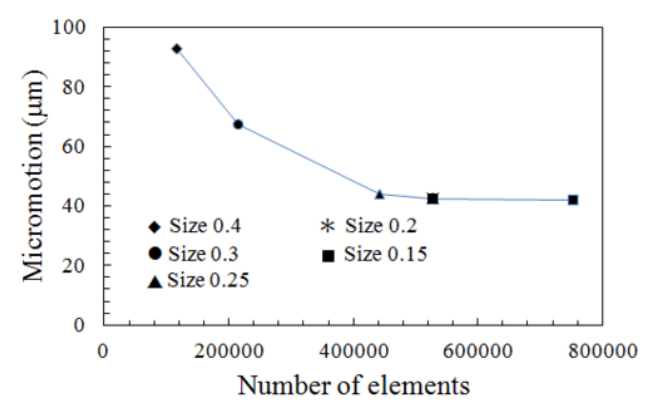

Fig. 7. Influence of the number of elements on micromotion in the finite element model.

value of micromotion is $43.02 \mu \mathrm{m}$, as shown in Figure 6 .

Figure 7 illustrates the influence of the number of elements on the micromotion of cancellous and cortical bones. Results indicate that when element size is smaller than $0.2 \mathrm{~mm}$, the distribution of micromotion is converged. Therefore, element size, a parameter that must be set during the analysis procedures, is set to $0.25 \mathrm{~mm}$.

\subsection{Factor analysis for new dental implant system}

Many endogenous and exogenous factors influence the success rate of dental implant systems. In material characteristic analysis, exogenous factors include: thread pitch (TP), thread depth (TD), larger diameter of the implant neck (D1), and smaller diameter of the implant neck (D2). Endogenous factors include the density of cancellous bone (DEN). These characteristics are assumed for the Zimmer dental implant system in this experiment.

Figure 8 presents the effect of thread pitch and thread depth on micromotion with various larger diameters of the implant neck. As shown in Figure 8(a), the micromotion decreased initially, and then increased with increasing thread pitch. Moreover, the micromotion evaluated using larger D1 diameters of the implant was generally higher than that obtained from smaller D1 diameters. As shown in Figure 8(b), the micromotion increased initially, and then decreased with increasing thread depth. The micromotion evaluated using the larger D1 diameter of the implant is also generally higher than that obtained from the smaller D1 diameters; however, the relationship is inverted as the thread depth rises above $0.4 \mathrm{~mm}$.

Figure 9 shows the effects of smaller diameter of the implant neck and the density of cancellous bone on micromotion with various larger diameters of the implant neck. As shown in Figure 9(a), the micromotion decreased initially, and then increased as the smaller diameter of the implant neck increased. In most cases, the micromotion evaluated using the larger D1 diameter of the implant was higher than that obtained from the smaller D1 diameters. As shown in Figure 9(b), the micromotion decreased initially and then increased as the density of cancellous bone increased. As shown in 


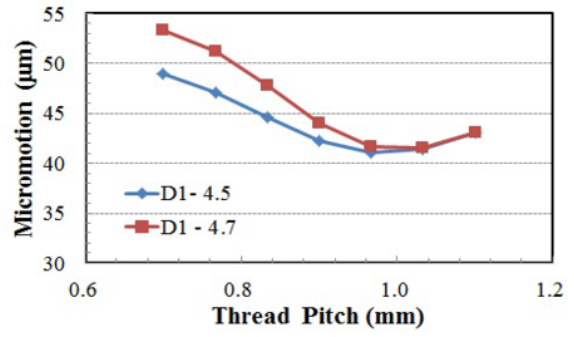

(a)

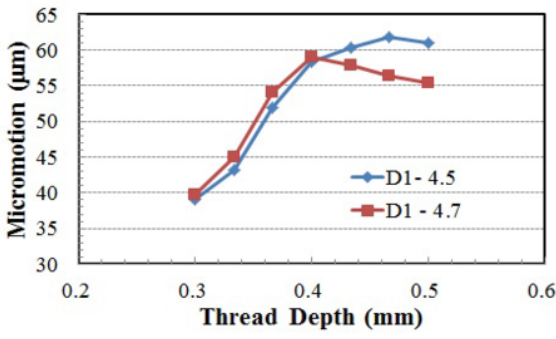

(b)

Fig. 8. Effects of (a) thread pitch and (b) thread depth on micromotion with various larger diameters of implant neck.

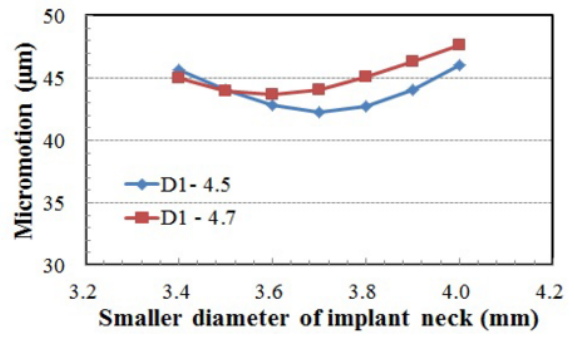

(a)

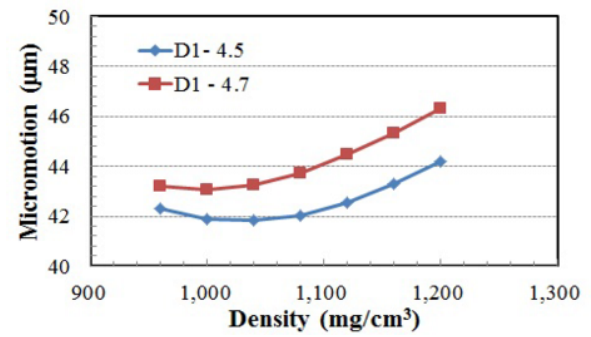

(b)

Fig. 9. Effects of (a) smaller diameter of implant neck and (b) density of cancellous bone on micromotion with various larger diameters of implant neck.

Table 3

Design ranges for control factors

\begin{tabular}{|c|c|c|c|c|}
\hline Control factor & Notation & Lower bound & Basic value & Upper bound \\
\hline Thread Pitch (mm) & TP & 0.7 & 0.9 & 1.1 \\
\hline Thread Depth (mm) & TD & 0.3 & 0.33 & 0.5 \\
\hline $\begin{array}{l}\text { Larger diameter of implant neck } \\
(\mathrm{mm})\end{array}$ & D1 & 4.2 & 4.5 & 4.8 \\
\hline $\begin{array}{l}\text { Smaller diameter of implant } \\
\text { neck }(\mathrm{mm})\end{array}$ & D2 & 3.4 & 3.7 & 4.0 \\
\hline Density $\left(\mathrm{mg} / \mathrm{cm}^{3}\right)$ & DEN & 960 & 1100 & 1200 \\
\hline
\end{tabular}

Figures 8 and 9, the micromotion evaluated using the larger D1 diameter was generally higher than that obtained using the smaller D1 diameter.

\section{Micromotion reduction and results}

As shown in Figure 1, five factors were chosen for analysis of the Zimmer dental implant system: thread pitch (TP), thread depth (TD), larger diameter of implant neck (D1), smaller diameter of implant neck (D2), and density of cancellous bone (DEN). The original values, lower bounds, and upper bounds of each control factor are given in Table 3. The design space is continuous due to the continuity of all factors; therefore, uniform design [8] can be applied to the experimental simulations. Uniform design has been widely used for optimization in many engineering applications $[9,10]$. Uniform design helps to select a set of sample points distributed homogeneously in the design 
space. For modeling data, the design range for each factor is segregated into several levels. According to the number of levels, the uniform table is utilized to design the experiments with the modeled data. As shown in Table 4, the uniform table consists of 12 rows and 10 columns [8]. Columns 1, 3, 4, 8 and 10 are selected, because there are five control factors in the new dental implant system [8]. Each experiment simulation of the Zimmer dental implant system is shown in Table 5. For each dental implant system, Pro/Engineer is utilized to construct the 3D models and ANSYS/LS-DYNA is employed to calculate the responses of the dental implant system under dynamic loads. Results indicate that minimum micromotion occurs during the ninth experiment; therefore, the dental implant design utilized in the ninth experiment determined to be the optimal design model. The improved edition of the implant model causes a micromotion of $36.42 \mu \mathrm{m}$, indicating that the primary implant stability of the full dental implant system has been reduced as the thread pitch and larger diameter of implant neck simultaneously increase.

\section{Conclusions}

Table 4

Uniform table

\begin{tabular}{lllllllllll}
\hline Experiment No. & 1 & 2 & 3 & 4 & 5 & 6 & 7 & 8 & 9 & 10 \\
\hline 1 & 1 & 2 & 3 & 4 & 5 & 6 & 8 & 9 & 10 & 12 \\
2 & 2 & 4 & 6 & 8 & 10 & 12 & 3 & 5 & 7 & 11 \\
3 & 3 & 6 & 9 & 12 & 2 & 5 & 11 & 1 & 4 & 10 \\
4 & 4 & 8 & 12 & 3 & 7 & 11 & 6 & 10 & 1 & 9 \\
5 & 5 & 10 & 2 & 7 & 12 & 4 & 1 & 6 & 11 & 8 \\
6 & 6 & 12 & 5 & 11 & 4 & 10 & 9 & 2 & 8 & 7 \\
7 & 7 & 1 & 8 & 2 & 9 & 3 & 4 & 11 & 5 & 6 \\
8 & 8 & 3 & 11 & 6 & 1 & 9 & 12 & 7 & 2 & 5 \\
9 & 9 & 5 & 1 & 10 & 6 & 2 & 7 & 3 & 12 & 4 \\
10 & 10 & 7 & 4 & 1 & 11 & 8 & 2 & 13 & 9 & 3 \\
11 & 11 & 9 & 7 & 5 & 3 & 1 & 10 & 8 & 6 & 2 \\
12 & 12 & 11 & 10 & 9 & 8 & 7 & 5 & 4 & 3 & 1 \\
\hline
\end{tabular}

Table 5

Experimental combinations and results

\begin{tabular}{lllllll}
\hline Experiment No. & TP $(\mathrm{mm})$ & TD $(\mathrm{mm})$ & $\mathrm{D} 1(\mathrm{~mm})$ & $\mathrm{D} 2(\mathrm{~mm})$ & $\mathrm{DEN}\left(\mathrm{mg} / \mathrm{cm}^{3}\right)$ & Micromotion $(\mu \mathrm{m})$ \\
\hline 1 & 0.70 & 0.34 & 4.36 & 3.84 & 1200.00 & 42.38 \\
2 & 0.74 & 0.39 & 4.58 & 3.62 & 1178.18 & 78.10 \\
3 & 0.77 & 0.45 & 4.80 & 3.40 & 1156.36 & 54.39 \\
4 & 0.81 & 0.50 & 4.31 & 3.89 & 1134.55 & 59.26 \\
5 & 0.85 & 0.32 & 4.53 & 3.67 & 1112.73 & 41.87 \\
6 & 0.88 & 0.37 & 4.75 & 3.45 & 1090.91 & 56.21 \\
7 & 0.92 & 0.43 & 4.25 & 3.95 & 1069.09 & 56.74 \\
8 & 0.95 & 0.48 & 4.47 & 3.73 & 1047.27 & 60.13 \\
9 & 0.99 & 0.30 & 4.69 & 3.51 & 1025.45 & 36.42 \\
10 & 1.03 & 0.35 & 4.20 & 4.00 & 1003.64 & 51.18 \\
11 & 1.06 & 0.41 & 4.42 & 3.78 & 981.82 & 37.92 \\
12 & 1.10 & 0.46 & 4.64 & 3.56 & 960.00 & 40.67 \\
\hline
\end{tabular}


This paper presented a systematic approach to improve the design of the Zimmer dental implant in order to reduce the maximum micromotion of an implant system under dynamic loads. For the original implant design, the micromotion of the Zimmer dental implant system was $43.02 \mu \mathrm{m}$; after uniform design improvements, the micromotion was reduced to $36.42 \mu \mathrm{m}$, demonstrating a $15.34 \%$ reduction in micromotion. Results indicate that uniform design is a powerful tool to reduce micromotion for the Zimmer implant model under dynamic loads. However, these finding have not modeled the fracture of bone, the effect of blood flow, or remodeling in order to preserve experimental simplicity. Therefore, limitations of this study include simplified homogeneous and isotropic material properties.

\section{Acknowledgment}

This research work was sponsored by research planes of the Ministry of Science and Technology of Taiwan, 103-2218-E-010-004 and 103-2221-E-327-018. The authors acknowledge gratefully their financial support.

\section{References}

[1] E. Javed and G.E. Romanos, The role of primary stability for successful immediate loading of dental implant: A literature review, Journal of Dentistry 38 (2010), 612-620.

[2] F. Isidor, Histological evaluation of peri-implant bone at implants subjected to occlusal overload or plaque accumulation, Clinical Oral Implants Research 8 (1997), 1-9.

[3] O. Kayabasi, E. Yüzbasioglu and F. Erzincanli, Static, dynamic and fatigue behaviors of dental implant using finite element method, Advances in Engineering Software 37 (2006), 649-658.

[4] E.S. Kim and S.Y. Shin, Influence of the implant abutment types and the dynamic loading on initial thread loosening, Journal of Advanced Prosthodontics 5 (2013), 21-28.

[5] S. Mammadzada, C. Artunç, F. Sen, M.A. Güngör, U. Tekin and E. Çömlekoğlu, Effect of abutment and implant shapes on stresses in dental applications using FEM, Mathematical and Computational Applications 16 (2011), 546-555.

[6] I.C. Chou, S.Y. Lee, M.C. Wu, C.W. Sun and C.P. Jiang, Finite element modelling of implant designs and cortical bone thickness on stress distribution in maxillary type IV bone, Computer Methods in Biomechanics and Biomedical Engineering 17 (2014), 516-526.

[7] P.J. Rubin, R.L. Rakotomanana, P.F. Leyvraz, P.K. Zysset, A. Curnier and J.H. Heegaard, Frictional interface micromotions and anisotropic stress distribution in a femoral total hip component, Journal of Biomechanic 26 (1993), 725-739.

[8] K.T. Fang and Y. Wang, Number-Theoretic Methods in Statistics, Chapman \& Hall, London, U.K., 1994.

[9] L. Peng, Y.Z. Wang, G.M. Dai and Z.S. Cao, A novel differential evolution with uniform design for continuous global optimization, Journal of Computers 7 (2012), 3-10.

[10] Y.Z. Yang, M. Jiang, J. Xu, Y.H. Ma and J. Tong, Uniform design of optimizing formulation of friction materials with composite mineral fiber (CMF) and their friction and wear behavior, Applied Composite Materials 19 (2012), 161-170. 